\title{
sciendo
}

ISSN: 1231-4005

e-ISSN: $2354-0133$

DOI: $10.2478 /$ kones-2019-0112

\section{MODELLING OF TRANSPORT SYSTEM OPERATIONAL RELIABILITY A MARKOV APPROACH}

\author{
Leszek Smolarek, Monika Ziemska \\ Gdynia Maritime University \\ Department of Transport and Logistics \\ Morska Street 81-87, 81-225 Gdynia, Poland \\ tel.: +48585586838 \\ e-mail:l.smolarek@wn.umg.edu.pl,m.ziemska@wn.umg.edu.pl
}

\begin{abstract}
The workability of a transport system is associated with performance and operational reliability. Operational reliability provides a measure of the probability that a transport system will realize transport process as intended. Performance reliability is an adequacy measure of transport process realization under specific environmental and traffic conditions. Transport system can be modelled as repairable, multistate, non-homogenous rectangular or dendrite system. This article provides the Markov and semi Markov models for estimation of the operational and performance reliability of city transport system. The system is semi homogenous it means that serial subsystems have the same reliability function. The reliability of any serial subsystem is exponential. The distribution of the repair time is any probability distribution. In case where the probability distribution of the repair time is exponential, the Markov process is used to construct simulation model. The simulation model was applied at Microsoft Excel. Many stochastic models in engineering, logistic and even finance or insurance are setup in a spreadsheet for simulation. The semi Markov model of the multistate reliability of repaired system is applied to the street system. The embedded Markov chain was used to count stationary probabilities. The possibility of application of the results is illustrated by an example for the systems with rectangular or dendrite shaped accordingly, consist of three types of elements.
\end{abstract}

Keywords: transport, road transport, simulation, Markov model, semi Markov model

\section{Introduction}

An efficiently operating transport system is the definition of a well-functioning city. Infrastructure connections between motion generators have an inherent impact on getting around the city. Reliability of communication routes is a definition of what period movement participants can transport from the starting point to the final point. Transportation systems play an important role in society, economic, national defence and others. They are classified as critical infrastructure systems because of the far-reaching effects of disruptions on these systems. Analysing the reliability on various elements of transportation systems such as infrastructure means of transportation were carried out since the mid-twentieth century $[5,6]$.

In the article, as a transport system, we mean part of a road or urban transport network. System states, due to its operational reliability, are determined by the level of traffic obstructions, that is:

- state " 0 " for level of service A and B,

- state "1" for level of service C,

- state "2" for level of service D and E,

- state "3" for level of service F.

In the case of modelling at the level of constituent communication lines, we assume that:

- the system is built of s series subsystems,

- the system has finite number of components,

- lifetime of any component $c$ of a series subsystem is exponential, with parameter $\lambda_{c}$,

- repair time has the distribution $G(t)$, with finite expected value equal $m_{r}$,

- life times of components and repair time are stochastically independent, 
- the components are repaired accordingly to the sequence they failed,

- only one component can be repaired at the same time

\section{The operational reliability Markov and semi-Markov models}

The system with finite or countable number of states can be described by semi-Markov process if it fulfils the following condition:

the probability that the system, which is in state $i$, goes to the state $j$ and will stay in this state for time $t_{0}$ is independent of the process history before the time $t$ and actual time $t$, [3].

Let $X(t)$ will be the stochastic process with finished or countable set of states $S=\{0,1, \ldots\}$, the beginning state $x_{0}$, Markov chain $X(n)$ - state of the process after $n$-th change and random variables $T_{k}$ the time in which the process $X(t)$ stay in state in state $k-1, k=1,2, \ldots$

The process $X(t)$ is the semi-Markov process, [2, 3,9] if for any moments $0 \leq t_{1}<$ $t_{2}, \ldots, t_{n-1}<t ; i, i_{1}, \ldots, k, j \in S$ and $n>1$, we have

$$
\begin{gathered}
P\left(\left\{T_{n}<t, X(n)=j / X(0)=i, T_{1}=t_{1}, \ldots, X(n-2)=k, T_{n-1}=t_{n-1}, X(n-1)=i\right\}\right) \\
=P\left(\left\{T_{n}<t, X(n)=j / X(n-1)=i\right\}\right)
\end{gathered}
$$

and

$$
P\left(\left\{T_{n}<t, X(n)=j / X(n-1)=i\right\}\right)=Q_{i j}(t), \text { for } n=1,2, \ldots
$$

Functions $Q_{i j}(t)$ can be represent as follows

$$
Q_{i j}(t)=p_{i j} F_{i j}(t)
$$

where

- the quantities $p_{i j}$ are the transitions probabilities of the embedded Markov chain $X(n)$,

- the functions $F_{i j}(t)$ are the conditional distributions that the process $X(t)$ stays in state $i$ if the next jump will be to the state $j$.

The operational reliability model of the system is shown in the graph Fig. 1.

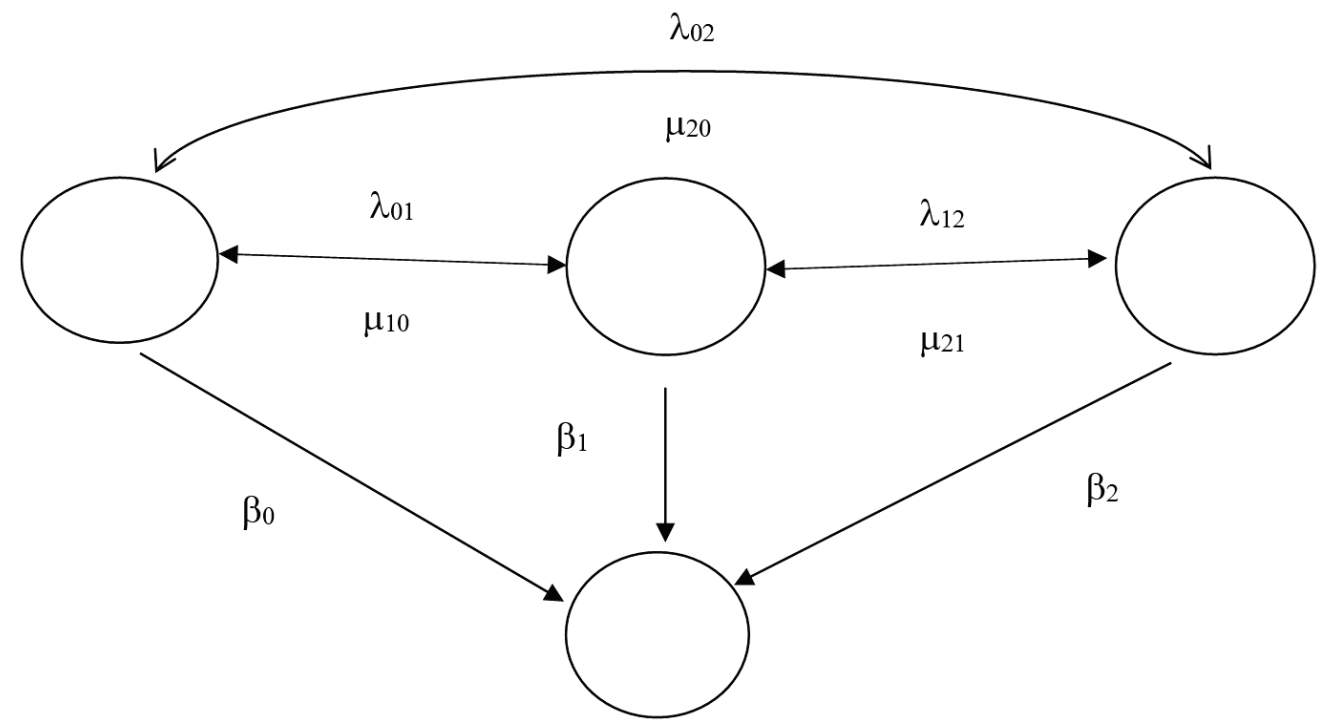

Fig. 1. A graph of the system operational reliability

Using the theorems presented at [3] we obtain that:

- if any $F_{i j}(t)$ is not the exponential distribution then the process $X(t)$ is not Markov,

- if all probability distribution are exponential then the process $X(t)$ is Markov,

- the set $\{0,1,2,3\}$ is the state space of the Markov chain of the process $X(t)$, 
- because the number of states is finite then there is the embedded ergodic Markov chain of the process $X(t)$,

- using the methods presented in [9], we have the formulas of stationary probabilities of embedded Markov chain,

$-p_{0}=1-\left(p_{1}+p_{2}+p_{31}\right)$.

The system of stationary equations describing the process of change of operational reliability states is in the form:

$$
\left\{\begin{array}{c}
-\lambda_{01} p_{0}-\lambda_{02} p_{0}+\lambda_{30} p_{3}+\lambda_{10} p_{1}+\lambda_{20} p_{2}=0 \\
-\lambda_{13} p_{2}-\lambda_{10} p_{1}-\lambda_{12} p_{1}+\lambda_{01} p_{0}+\lambda_{20} p_{2}=0 \\
-\lambda_{23} p_{2}-\lambda_{20} p_{2}-\lambda_{21} p_{2}+\lambda_{02} p_{0}+\lambda_{21} p_{2}=0 \\
-\lambda_{30} p_{3}+\lambda_{03} p_{0}+\lambda_{13} p_{1}+\lambda_{23} p_{2}=0
\end{array}\right.
$$

where:

$p_{k}$ - indicate the limit probability for the state $k, k=0,1,2,3$,

$\lambda_{j k}$ - means the intensity of the transition from state $\mathrm{j}$ to state $k, j, k=0,1,2,3$.

By replacing the last equation with the normalizing condition, (1), we obtain the matrix equation of the form, $[3,4]$ :

$$
\left[\begin{array}{cccc}
-\lambda_{01}-\lambda_{02} & \lambda_{10} & \lambda_{20} & \lambda_{30} \\
\lambda_{01} & -\lambda_{13}-\lambda_{10}-\lambda_{12} & \lambda_{20} & 0 \\
\lambda_{02} & \lambda_{21} & -\lambda_{23}-\lambda_{20}-\lambda_{21} & 0 \\
1 & 1 & 1 & 1
\end{array}\right]\left[\begin{array}{l}
p_{0} \\
p_{1} \\
p_{2} \\
p_{3}
\end{array}\right]=\left[\begin{array}{l}
0 \\
0 \\
0 \\
1
\end{array}\right] .
$$

Assuming that the ratio of the intensity of $\lambda_{02}$ to the intensity of $\lambda_{01}$ is $p$ and the remaining intensities are equal, we obtain from the system of equations (2), [7]:

$$
p_{0}=\frac{1}{2+p}, p_{1}=\frac{1}{2(2+p)}, p_{2}=\frac{1+3 p}{8(2+p)}, p_{3}=\frac{1}{2+p} \text {. }
$$

\section{Example 1. Markov model, three reliability states}

Consider the system where all probability distribution are exponential and a system can pass through only three states: 0 (full operational reliability), 1 (reduced operational reliability) and $2 \& 3$ (complete operational unreliability-failure). In spite of the simplicity of the model, remarkable results are obtained concerning the behaviour of the system according traffic changes. The transition graph for the process is shown in Fig. 2, where:

- 0 - state of full operational reliability,

- 1 - state of reduced operational reliability,

- $2 \& 3$ - state of complete operational unreliability (failure).

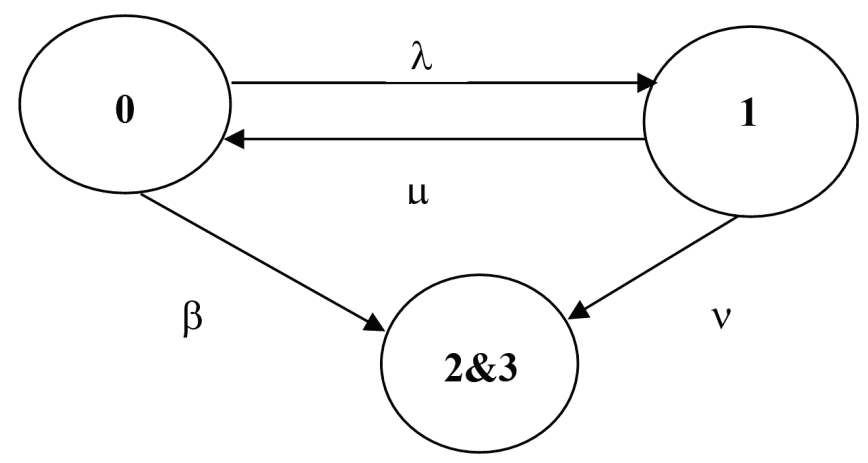

Fig. 2. Transition graph of the system operational reliability model, Markov three state case

The Chapman-Kolmogorov forward equations are given by: 


$$
\begin{gathered}
p^{\prime}{ }_{0}(t)=-(\lambda+\beta) p_{0}(t)+\mu p_{1}(t), \\
p^{\prime}{ }_{1}(t)=-(\mu+v) p_{1}(t)+\lambda p_{0}(t), \\
p^{\prime}{ }_{2 \& 3}(t)=\beta p_{0}(t)+v p_{1}(t)
\end{gathered}
$$

and the operational reliability function $R_{o r}(t)$, according (4), is

$$
R_{\text {or }}(t)=1-\int_{0}^{t}\left[\beta p_{0}(t)+v p_{1}(t)\right] d \tau
$$

We assume that at time zero system is in state 0 . Using the Laplace transform methods to equations (4-5), we have:

$$
\begin{gathered}
p_{0}(t)=\frac{s_{1}+\mu+v}{s_{1}-s_{2}} e^{s_{1} t}-\frac{s_{2}+\mu+v}{s_{1}-s_{2}} e^{s_{2} t} \\
p_{1}(t)=\frac{\lambda}{s_{1}-s_{2}} e^{s_{1} t}-\frac{\lambda}{s_{1}-s_{2}} e^{s_{2} t} \\
p_{2 \& 3}(t)=1+\frac{\left(s_{1}+\mu+v\right) \beta+\lambda v}{\left(s_{1}-s_{2}\right) s_{1}} e^{s_{1} t}-\frac{\left(s_{2}+\mu+v\right) \beta+\lambda v}{\left(s_{1}-s_{2}\right) s_{2}} e^{s_{2} t},
\end{gathered}
$$

where:

$$
\begin{aligned}
& \mathrm{s}_{1}=\frac{1}{2}\left[-(\lambda+\beta+\mu+v)-\sqrt{1+\frac{4 \lambda \cdot \mu}{(-\lambda-\beta+\mu+v)^{2}}} \cdot(-\lambda-\beta+\mu+v)\right], \\
& \mathrm{s}_{2}=\frac{1}{2}\left[-(\lambda+\beta+\mu+v)+\sqrt{1+\frac{4 \lambda \cdot \mu}{(-\lambda-\beta+\mu+v)^{2}}} \cdot(-\lambda-\beta+\mu+v)\right] .
\end{aligned}
$$

\section{Example 2. Semi-Markov model, four reliability states, simulation approach}

We suppose, that $P(0), P(1), P(2), P(3)$ mean the probability of being in the respective states of the system operational reliability model, Fig. 1.

We define the stochastic process $\left(i_{n}, T_{i_{n} i_{n+1}}\right)$ is a non-homogeneous Markovian renewal process, where ([2-5]):

$n$ - number of the iterations, $n \in N$,

$\mathrm{S}$ - the set of states,

$T_{i j}$ - the random variable represents the transition's time between the states $i$ and $j$,

$i_{n}-\mathrm{a}$ random variable with the set of states: $\mathrm{S}=\left\{\mathrm{S}_{\mathrm{B}}, \mathrm{S}_{\mathrm{ZB}}, \mathrm{S}_{\mathrm{NB}}, \mathrm{C}\right\}$ representing the state at the $n$-th transition,

$\left\{i_{n}: n \in N\right\} \quad$ - the sequence of the realizations of the embedded non-homogeneous Markov chain, $\left\{\xi_{n}: n \in N\right\}$

$\left\{T_{i_{n} i_{n+1}}: n \in N\right\}$ - a sequence of the independent random variables which represents the time of the transition from state $i_{n} \in \mathrm{S}$ to $i_{n+1} \in \mathrm{S}$,

$\mathbf{P}(t)=\left[p_{i j}(t)\right]$ - the transition matrix at the time $t$ of the embedded non-homogeneous Markov chain.

$$
\mathbf{P}=\left[\begin{array}{cccc}
0 & p_{12}(t) & p_{13}(t) & 0 \\
p_{21}(t) & 0 & p_{23}(t) & p_{24}(t) \\
0 & p_{32}(t) & 0 & p_{34}(t) \\
0 & 0 & 0 & 1
\end{array}\right]
$$


Let assume that $i \in \mathrm{S}$ is the beginning state of the Semi-Markov process. The next state of the process, $j \in \mathrm{S}$, is obtained by the draw according to the distribution given in $i$-th line of the matrix $\mathbf{P}$, i.e. based on the following formulae, [3]:

$$
j=\min \left\{r: y_{i r-1}<u \leq y_{i r}\right\},
$$

where:

$y_{i j}=\sum_{a=0}^{j} p_{i a}, \quad p_{i 0}=0$.

It is possible to generate the realizations of the independent random variable $T_{i j}$ by transforming the realizations of the copies the random variables $u$ with uniform distribution. Based on this fact, the compound ([3]):

- for the exponential distribution is given as:

$$
T_{i j}=-\frac{1}{\lambda} \ln (1-u)
$$

- for the Weibull distribution is given by:

$$
T_{i j}=\left(-\frac{1}{\lambda} \ln (1-u)\right)^{\frac{1}{\alpha}},
$$

where $u$ is the random variable with uniform distribution $\mathrm{U}(0,1), \lambda>0, \alpha>0$.

Algorithm for finding of the characteristics of the system's operational reliability:

- chose the beginning state $i$,

- generate the realization the random variable $\mathrm{u}$ with uniform distribution $u(0,1)$,

- determine the state $\mathrm{j}$ according to formulas,

- generate realization of the random variable $t_{i j}$ using the formulas (11-12),

- evaluate the time to the moments to achieve the 2 state,

- evaluate the time to the moments to achieve the 3 state,

- estimate the characteristics such that histogram, the mean values, the second moments and the standard deviations.

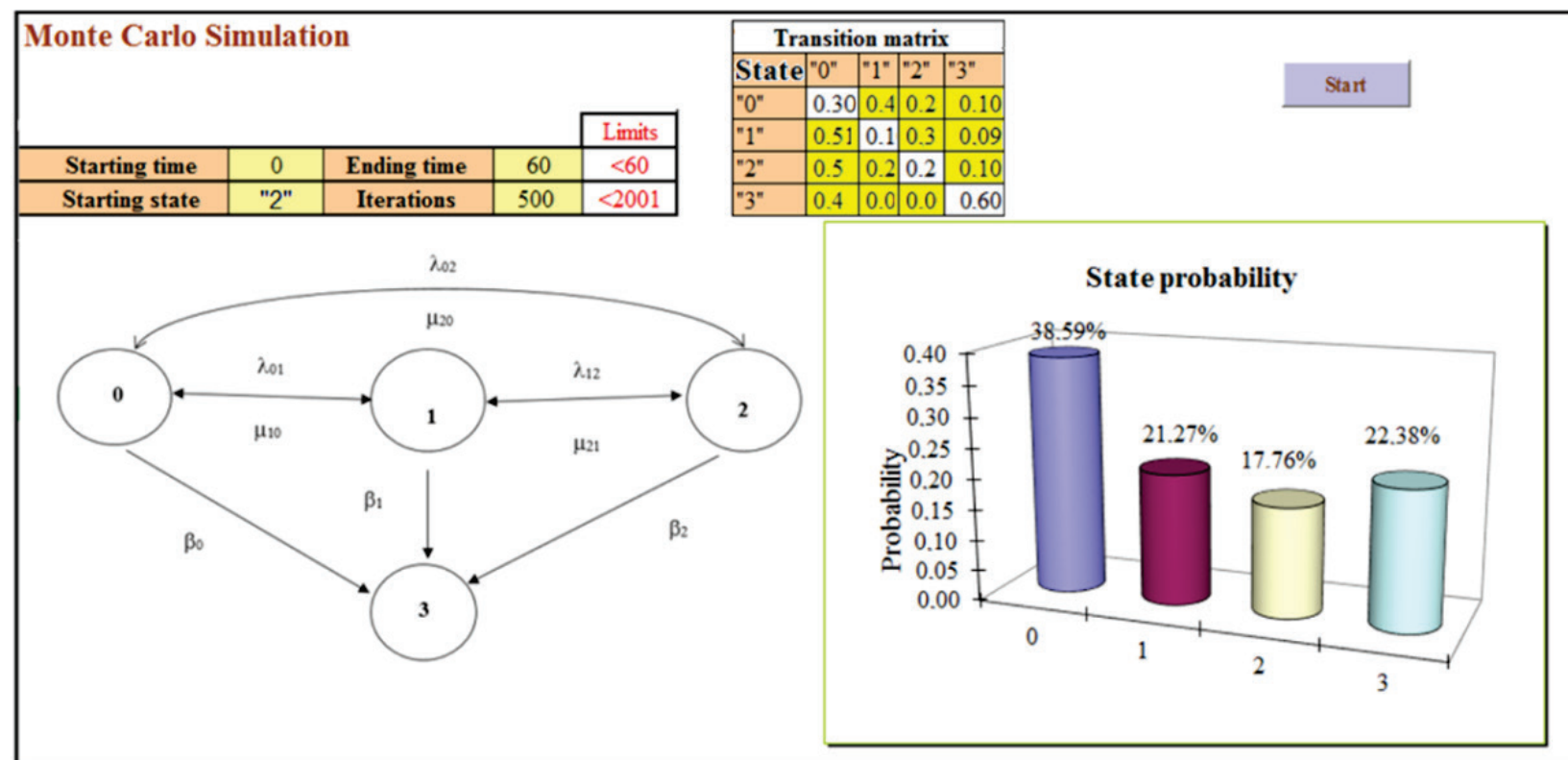

Fig. 3. An example of simulation results, starting state " 2 " 


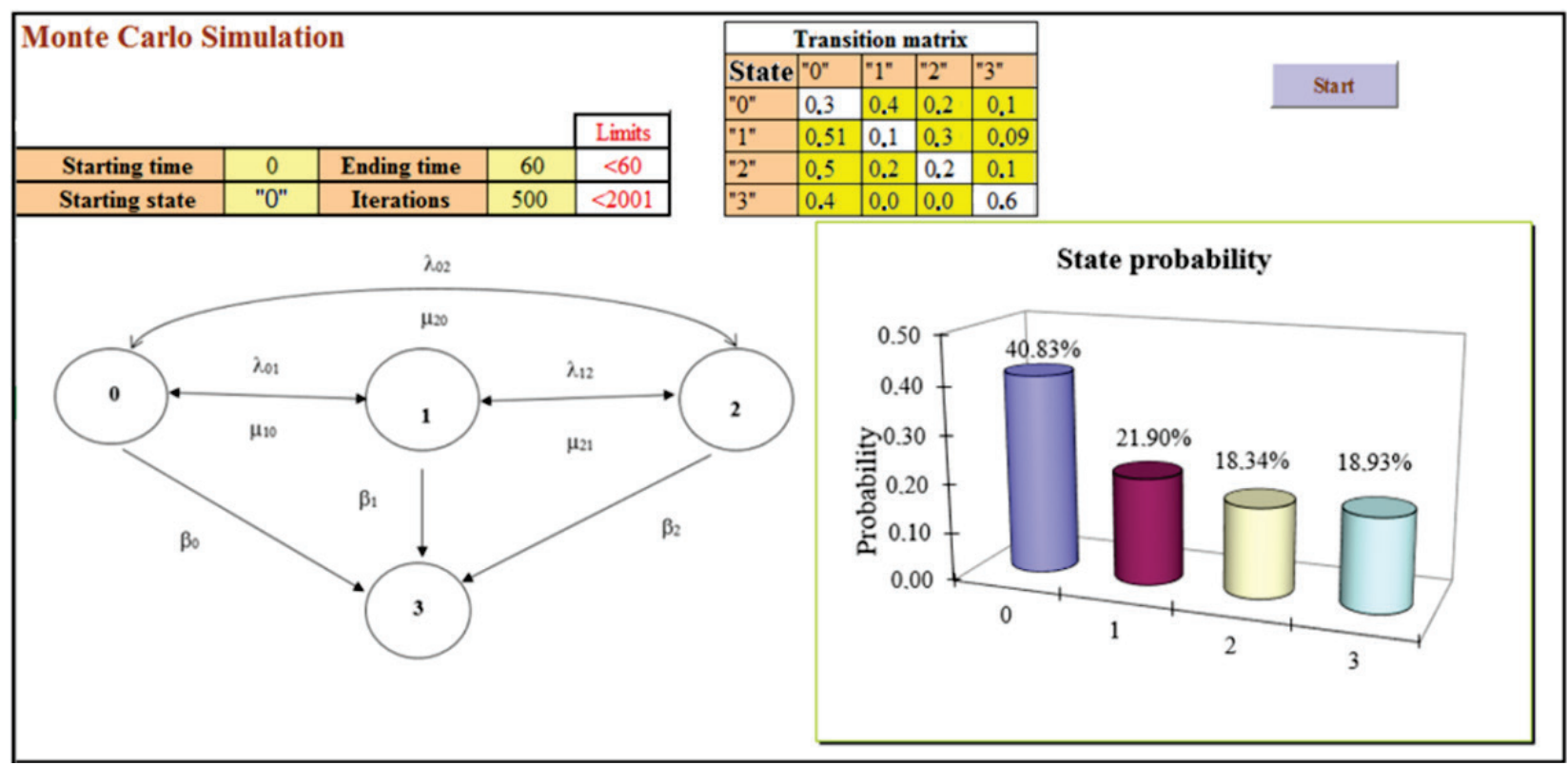

Fig. 4. An example of simulation results, starting state "0"

Example 3. Semi-Markov model, rectangular system

The system is built of parallel series subsystems connected on the input and output ends. All series subsystems have the same type of reliability function with finite expected value (the serial subsystems are homogenous). The state of the system is equal to the number of not broken series subsystems. The examples of such system are shown at Fig. 1 and Fig. 2

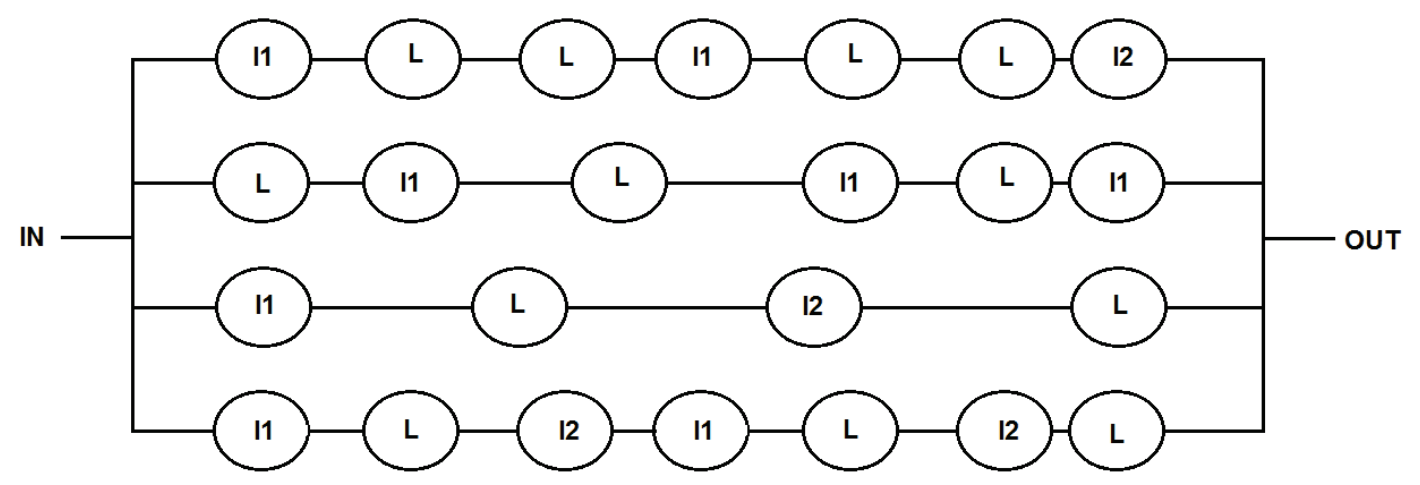

Fig. 5. A rectangular example of the system model

where:

- "I1" intersection type 1,

- "I2" intersection type 2,

- ..............................,

- "L" section between intersections.

We assume that:

- the system is in state $k$ if only $k$ series subsystems are in workable state and others are broken,

- $X(t)$ - the number of working series subsystems (components) at time $t$,

$\boldsymbol{P}=\left(P_{0}, P_{1}, \ldots, P_{s}\right)-$ stationary, ergodic probabilities of stochastic process $X(t)$,

- $t_{n}$ - the moment in which the $n$-th repair is ended,

- $\zeta_{n}$ - the number of working series subsystems before the time $t_{n}$, it is the embedded Markov chain of the process $X(t)$,

$-\widehat{\boldsymbol{p}}=\left(\hat{p}_{0}, \hat{p}_{1}, \ldots, \hat{p}_{s}\right)$ - stationary probabilities of embedded Markov chain $\zeta_{n}$. 
Because the finite set $\{0,1,2, \ldots, s-1, s\}$ is the state space of the process $\zeta_{n}$ then the process is ergodic.

The homogenous Markov chain $\zeta_{n}$ has the transition probabilities given by formulas

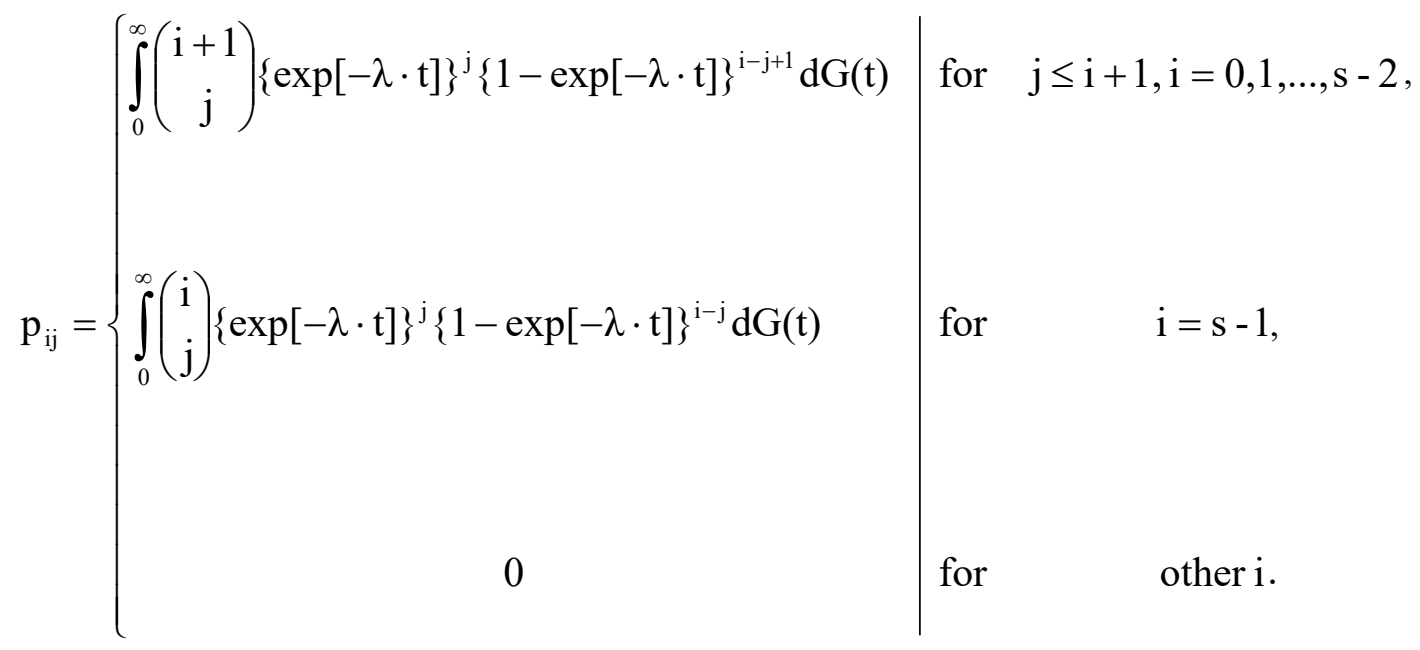

The stationary probabilities $\widehat{\boldsymbol{p}}=\left(\hat{p}_{0}, \hat{p}_{1}, \ldots, \hat{p}_{s}\right)$ of embedded Markov chain $\zeta_{n}$ can be find using the following formulas, $[3,7,8]$ :

$$
\begin{gathered}
\hat{p}_{0}=1-\sum_{k=1}^{s} \hat{p}_{k} ; \hat{p}_{k+1}=\frac{s \hat{p}_{k}}{(k+1)\left(s \lambda m_{r}+\hat{p}_{s-1}\right)}, \\
\hat{p}_{k}=\sum_{i=1}^{s}(-1)^{i-1}\left(\begin{array}{c}
i \\
k
\end{array}\right) \frac{s C_{i-1}}{i} \frac{\sum_{j=i-1}^{s-1}\left(\begin{array}{c}
s-1 \\
j
\end{array}\right) C_{j}^{-1}}{\left(1+s m_{r} \lambda\right) \sum_{j=0}^{s-1}\left(\begin{array}{c}
s-1 \\
j
\end{array}\right) C_{j}^{-1}} ; C_{0}, C_{j}=\prod_{w=1}^{j} \frac{G^{*}\left(w m_{r}\right)}{1-G^{*}\left(w m_{r}\right)},
\end{gathered}
$$

where: $k=0,1, \ldots, s$.

\section{Summary}

The models of reliability of the system operation presented in the article can be used for estimation and forecasting in everyday practice. The methods can be useful for estimating selected operational reliability characteristics of the urban transport system. It is possible to extend the results to systems with other complex reliability structures. It is possible to obtain meticulous results using the above-described model based on input data such as traffic intensity, intensity on torsional relations, duration of green light (in the case of intersections with traffic lights), etc. The calculations presented in the article may be an indispensable component in the aspect of intelligent transport systems and more specifically modules related to event detection. The use of mathematical solutions is indispensable in defining limit states where urban traffic is defined by the free speed of moving vehicles in the urban transport network defined by a given infrastructure sequence.

\section{References}

[1] Ionescu, D. C., Limnios, N., (eds), Statistical and Probabilistic Models in Reliability, pp. 153-183, Birkhauser, Boston 1998.

[2] Guan, Y., Li, S. E., Duan, J., Wang, W., Cheng, B., Markov probabilistic decision making of self-driving cars in highway with random traffic flow: a simulation study, Journal of Intelligent and Connected Vehicles, 1, 10.1108/JICV-01-2018-0003, 2018.

[3] Korolyuk, V. S., Turbin, A. F., Semi-Markov Processes and Their Applications, Naukova Dumka, in Russian, Kiev 1976. 
[4] Korolyuk, V. S., Korolyuk, V. V., Stochastic Models of Systems, Kluver Academic Publishers, 1999.

[5] Chryssaphinou, O., Limnios, N., Malefaki, S., Multi-State Reliability Systems Under Discrete Time Semi-Markovian Hypothesis, Reliability, IEEE Transactions, No. 60, 80-87, 10.1109/ TR.2010.2104210, 2011.

[6] Restel, F. J., Reliability and safety models of transportation systems - A literature review, PSAM 2014 - Probabilistic Safety Assessment and Management, 2014.

[7] Smolarek, L., The reliability models of large scale rectangular Weibull multistate systems, Proc. ESREL'99, Safety and Reliability, Vol. 1999, pp. 85-91, 1999.

[8] Smolarek, L., A Semi Markovian Model of Multistate Dendrite System, Mathematical Methods in Reliability, Statistique Mathematique et ses Applications, Bordeaux 2000, Vol. 2, pp. 973-977, France 2000.

[9] Takacs, L., Introduction to the theory of queues, Oxford University Press, Oxford 1962. Manuscript received 08 July; approved for printing 22 November 2019 